\title{
De normas, tendencias y otras regularidades en traducción audiovisual
}

\author{
JuAn José Martínez Sierra \\ Universidad de Murcia \\ sierra@um.es
}

Recibido: 10 de marzo de 2010

Aceptado: 2 de noviembre de 2010

\section{RESUMEN}

En este artículo se parte del marco de la escuela de la manipulación para ofrecer un completo resumen crítico de la teoría de las normas, prestando especial atención al ámbito de la traducción audiovisual. Por otro lado, se propone reflexionar acerca de si las actitudes puramente descriptivas en investigación pueden continuar de manera indefinida o si está próximo el momento de acompañar dichas posiciones con comportamientos prescriptivos. Por último, se presenta el término tendencia no sólo como paso intermedio entre una estrategia y una norma, sino también como una útil herramienta de trabajo en la investigación centrada en la búsqueda de regularidades traductoras.

Palabras clave: tendencia, norma, descripción, prescripción, traducción audiovisual.

On norms, tendencies and other regularities in audiovisual translation

\begin{abstract}
In this article reference to the manipulation school is made to offer a complete and critical summary of the norm theory, paying particular attention to the field of audiovisual translation. Besides, a reflection is proposed on whether purely descriptive attitudes in research can continue in an indefinite way or the moment for prescriptive behaviors has arrived. Finally, the term tendency is suggested not only as an intermediate step between a strategy and a norm, but also as a useful tool to carry out research centred on the search for translational regularities.
\end{abstract}

Key words: tendency, norm, description, prescription, audiovisual translation.

Sumario: 1. La escuela de la manipulación y la teoría de las normas. 2. El concepto de norma. 2.1. Origen del concepto. 2.2. Las normas como constructo social. 2.3. Características de las normas. 2.4. Clases de normas de traducción. 2.4.1. Las normas de Toury. 
2.4.2. Crítica al modelo de Toury y otras propuestas. 2.5. Normas en traducción audiovisual. 3. Tendencias de traducción. 4. Precisiones finales.

\section{La escuela de la manipulación y la teoría de las normas}

Iniciaremos nuestra discusión con una breve referencia a los estudios descriptivos, para después centrarnos en la teoría de las normas, algo que tiene sentido hacer dado que dicha teoría se desarrolla en el seno del citado modelo. La primera precisión que debemos hacer tiene que ver con el hecho de que los estudios descriptivos son objeto de cierta diversidad terminológica, ya que se trata de un paradigma también conocido como escuela de la manipulación o teoría del polisistema, si bien, como indica Marco (2002: 25), los especialistas parecen coincidir en denominar al paradigma con la etiqueta escuela de la manipulación (pese a que algunos no lo consideren una escuela en sentido estricto $)^{1}$.

Con objeto de resumir de forma completa y representativa las ideas principales que quisiera trasladar sobre la escuela de la manipulación bastará con rescatar la siguiente cita de Hermans (1985: 10-11):

The group is not a school, but a geographically scattered collection of individ-uals with widely varying interests, who are, however, broadly in agreement on some basic assumptions - even if that agreement, too, is no more than relative, a common ground for discussion rather than a matter of doctrine. What they have in common is, briefly, a view of literature as a complex and dynamic system; a conviction that there should be a continual interplay between theoretical models and practical case studies; an approach to literary translation which is descriptive, target-oriented, functional and systemic; and an interest in the norms and constraints that govern the production and reception of translations, in the relation between translation and other types of text processing, and in the place and role of translations both within a given literature and in the interaction between literatures.

Por otra parte, y como también recoge Marco (2002: 33-36), se trata de un modelo que presenta junto a las virtudes que se desprenden de dicha cita una serie de defectos como, por ejemplo, no poder cubrir por completo el campo de los estudios sobre la traducción literaria o el escaso interés que muestra por la lingüística ${ }^{2}$. Auto-

${ }^{1}$ Es interesante el repaso que hace Marco (2002) del estado de la cuestión previo a la aparición de la rama descriptiva, así como de la propia teoría.

${ }^{2}$ La escuela de la manipulación surge inicialmente para ocuparse de los asuntos propios de la traducción literaria. Sin embargo, no han faltado tampoco los autores que han aplicado este enfoque al ámbito de la traducción audiovisual aunque, como señala Cattrysse (1994: 43), no fue hasta finales de los ochenta cuando este modelo se empezó a aplicar a dicho campo. Como se verá, uno de los autores que mayor interés ha despertado en este aspecto es Karamitroglou (2000), quien dedica su trabajo a la cuestión de la investigación de las normas en el marco de la traducción audiovisual. 
res como Díaz Cintas (2005: 17) señalan incluso la contradicción que supone el deseo de evitar ser prescriptivo en prácticas como la subtitulación, en la que la propia presentación de los subtítulos supone un inevitable grado de prescriptivismo. Este autor no obvia tampoco en sus críticas el hecho de que el modelo evite evaluar y analizar los errores de traducción, lo que a su juicio, además de reducir posibles vías investigadoras, lo aleja del ámbito docente, abriendo así una brecha entre docencia e investigación. De modo similar, se me ocurre que cabría cuestionarse dónde está la línea entre descriptivismo y prescriptivismo. Es decir, ¿hasta qué punto puede el investigador limitarse a contemplar de manera estoica soluciones fallidas? ¿Puede avanzar la disciplina audiovisual sin voces que, además de describir qué ocurre (práctica actualmente extendida), indiquen qué hay que hacer para mejorar aquello que se describe ${ }^{3}$ ? En cualquier caso, no será aquí donde se acometa tal reflexión, ya que entiendo que ello podría suponer cuestionar la teoría objeto del presente trabajo, algo que sobrepasaría sin duda los límites marcados y que dejo, por tanto, para futura reflexión (¿podría plantearse una línea mixta, quizá?).

Respecto a la teoría de las normas hay que precisar que, si bien es cierto que se trata de una de las teorías desarrolladas dentro del paradigma descriptivista, ambos modelos no son sinónimos. Dicho de otro modo, no se debe reducir el enfoque descriptivista a la teoría de las normas. Es más, tampoco han de confundirse ambos conceptos, ya que el descriptivismo constituye una metodología de trabajo, mientras que la teoría de las normas es precisamente eso, una teoría ${ }^{4}$.

Según comenta Hermans (1999: 73), la perspectiva descriptiva considera las normas como objetos de estudio. Dicha perspectiva pretende teorizar y analizar la naturaleza de éstas y el modo en que funcionan y afectan a la práctica de la traducción. Sin embargo, no se propone establecer reglas, normas o pautas respecto al modo en el que el traductor debe proceder, algo que supondría una actitud prescriptiva.

He hablado de la orientación descriptiva del modelo que nos ocupa como uno de los rasgos definitorios del mismo. En su momento, Holmes dividió la disciplina de los Estudios sobre la Traducción en las ramas pura (teórica y descriptiva) y aplicada. En su esquema de 1972, el autor subdividió la subdisciplina descriptiva en tres clases: los estudios orientados hacia el producto, los orientados hacia la función y los orientados hacia el proceso. Críticas aparte, la formulación del concepto de norma se deriva desde el estudio del proceso o, más concretamente, de las decisiones y de las restricciones que caracterizan al proceso, según comenta Ballester (2001: 15). Por mi parte, no concibo la orientación hacia el producto o hacia el proceso como dos extremos excluyentes, ya que opino que existe un continuo entre ambos conceptos. Un trabajo descriptivo no ha de limitarse al estudio del resultado

\footnotetext{
3 Parece ser que esta pregunta va tomando consistencia. De hecho, ya se han puesto en marcha algunos proyectos con un cierto cariz prescriptivo, como por ejemplo Digital Television for All, financiado por la Unión Europea. Dicho proyecto pretende, entre otras cuestiones, evaluar la calidad de los subtítulos para luego ofrecer algunas recomendaciones (para más información, véase Romero Fresco, 2009).

4 De ahí que se pudiera argumentar que Toury confunde teoría y metodología.
} 
(producto) del proceso traductor (es decir, a la traducción en sí), sino que también se puede ocupar del proceso y, dentro de éste, prestar especial atención a las decisiones tomadas por el traductor/a (o equipo de traductores/as) de, por ejemplo, una serie de televisión, e incluso a las restricciones propias de la actividad.

Como afirma Marco (2002: 29-30), los seguidores de la escuela de la manipulación han abogado por la necesidad de describir la realidad en lugar de pretender influir en ella o cambiarla, sin que ello, como bien apunta Toury (1995: 15-19), sea óbice para que no exista una relación entre teoría y descripción o para que las conclusiones obtenidas a partir de la descripción y de la explicación no sean de utilidad en la rama aplicada de los Estudios sobre la Traducción. El objetivo es, pues, describir y explicar los hechos y, a partir de la observación empírica y de sus resultados, formular leyes generales sobre la conducta traductora. En mi opinión, es quizás aquí donde de nuevo las fronteras entre descripción y prescripción se muestran borrosas, puesto que podría interpretarse que la formulación de dichas leyes responde a una actitud prescriptiva, algo que resultaría paradójico. En todo caso, si consideramos las traducciones como productos de una determinada cultura, podemos investigar las razones por las que se ha optado por unas opciones y no por otras, atendiendo también a las condiciones bajo las que se ha llevado a cabo dicha selección (trabajos como el de Veiga, 2006, tratan de ofrecer una mejor comprensión de la esencia de las elecciones traductoras en el marco de la traducción del humor de tipo audiovisual). Cuando dicha observación haga patente la existencia de regularidades de comportamiento, será posible considerar que ciertas normas establecidas en la cultura meta han gobernado la labor traductora. Como veremos más adelante, a lo mejor cabría preguntarse en qué momento dichas regularidades dejan de serlo y se convierten en normas.

Desde la perspectiva audiovisual, Karamitroglou (2000: 14) nos recuerda que los defensores del paradigma descriptivista consideran los Estudios sobre la Traducción como una ciencia normativa (normativa no de una forma prescriptiva sino, como aclara Chesterman, 1993, en el sentido descriptivo de ciencia que estudia las normas). El autor nos cuenta también que el término norma se acuñó para describir un amplio abanico de fenómenos sociales que siguen un determinado patrón, y no sólo para ocuparse de los fenómenos lingüísticos o de traducción. Sobre esto último, Marco (2002: 32-33) precisa que, pese haberse revelado como un elemento clave y productivo de la rama descriptiva de los Estudios sobre la Traducción, el concepto de norma no es exclusivo de dichos estudios. Se trata de una noción común en el campo general de las ciencias humanas y sociales, en especial de la Psicología.

Karamitroglou (2000: 14) añade que la traducción audiovisual parece estar particularmente dominada por una fuerte presencia de normas debido al impacto de los medios de comunicación de masas en el público ${ }^{5} \mathrm{y}$ al hecho de que, como Ivarsson (1992: 66) comenta, los espectadores son criaturas de hábitos. Se trata, como bien determina Karamitroglou, de un ámbito en el que las normas actúan, además de sobre las decisiones que se toman al más bajo nivel textual, sobre las decisiones que se toman más allá de dicho nivel.

\footnotetext{
5 Véase Martínez Sierra (2008a).
} 
Pese a todo lo expuesto, entiendo que el hecho de que se recurra a la teoría de las normas con el fin de sustentar teóricamente un trabajo descriptivo no debe ser interpretado como que dicha teoría es capaz de explicar todo lo que ocurre en traducción. Su valor, pues, ha de ser considerado en su justa medida y con precaución.

Definamos, pues, el concepto de norma y repasemos las diferentes clases de normas que se han detectado tanto en el marco general de la traducción como en el ámbito particular de la traducción audiovisual.

\section{El concepto de norma}

\subsection{Origen del concepto}

Como podemos leer en la obra de Hermans (1999: 73-74), el modelo generativo de Levý (1967) caracterizó la traducción como un proceso de toma de decisiones que enfatizaba el hecho de que, en cada nivel, el traductor debe escoger una opción entre una serie de alternativas sabiendo que cada decisión afectará a las siguientes. Por otra parte, el conjunto de decisiones y de alternativas consideradas y desechadas es lo que determina la forma final del texto. En definitiva, el interés de Levý por la toma de decisiones subraya el poder y la responsabilidad del traductor.

A partir de lo anterior, y asumiendo por tanto la existencia de una serie de decisiones que el traductor toma y que no están totalmente predeterminadas ni son completamente idiosincrásicas, Hermans se pregunta qué es lo que conduce al traductor a optar por ciertas opciones y no por otras, y además a hacerlo no de forma puntual, sino regular. El autor encuentra la respuesta a su pregunta en Popovič (1970: 79), quien, refiriéndose a Levý, comenta que la traducción supone una confrontación de dos juegos de normas y convenciones lingüísticas y discursivas: por un lado, aquellas que residen en el texto origen y, por otro, aquellas que prevalecen en la cultura meta (o en una sección de ésta). Según lo interpreta Hermans, en los casos en los que es posible elegir de manera libre, el traductor se decidirá por una opción, y no por otra, por estar al tanto de, y en respuesta a, ciertas preferencias y expectativas que sabe existen en la cultura meta. La regularidad en la toma de dichas decisiones en distintos textos facilitará el establecimiento (en sentido no-prescriptivo) de patrones que, a su vez, afectarán a las expectativas de los receptores de textos traducidos. Es de esta manera como se fijan las normas las cuales, pues, son parte de la respuesta de por qué un traductor tiende a tomar ciertas decisiones en lugar de otras. En este punto, no podemos ignorar trabajos como el de Fawcett (2003), quien, a partir de los resultados de su estudio, reivindica la necesidad de no obviar que, en traducción audiovisual, al igual que en otras variedades traductoras, hay espacio para la aleatoriedad humana, oponiéndose así a aquellos que defienden el concepto de la invisibilidad del traductor como clave en la práctica traductora occidental.

A finales de los años setenta, Toury introdujo la noción de norma para referirse a las regularidades observadas en la conducta traductora dentro de una determinada situación sociocultural, noción que desde entonces ha venido influyendo en gran parte del trabajo realizado. La obra de este autor (inscrita bajo la etiqueta de los 
Estudios descriptivos sobre la traducción o Descriptive Translation Studies) deriva de los planteamientos de la escuela de la manipulación. Como Baker (1998: 163) recoge, el interés de Toury (inspirándose en el trabajo de Even-Zohar) reside principalmente en realizar afirmaciones acerca de aquello en lo que la conducta traductora consiste, y no sobre aquello en lo que ésta debería consistir; en otras palabras, describir, no prescribir. Con el fin de que dichas afirmaciones se muestren de manera sistemática y de que no constituyan una selección aleatoria de observaciones, éstas deberán mostrarse en forma de generalizaciones que sean aplicables a una clase o subclase particular de fenómenos y que se puedan testar intersubjetivamente (es decir, objetivamente entre varios sujetos). La noción de norma, pues, proporciona a Toury una categoría descriptiva que le permite realizar esas afirmaciones noaleatorias y verificables sobre tipos de comportamientos traductores.

Con objeto de entender la noción que nos ocupa, se hace necesario considerar el lugar en el que Toury ubica las normas. Para el autor (1995: 54), las restricciones socioculturales se han descrito a lo largo de una escala en uno de cuyos polos encontramos reglas generales y relativamente absolutas, mientras que en el otro hallamos idiosincrasias puras. En el amplio espacio comprendido entre ambos extremos se sitúan una serie de factores intersubjetivos que comúnmente se designan como normas, que a su vez forman un continuo a lo largo de la escala, siendo difusos los bordes existentes entre los distintos tipos de restricciones. Partiendo de este planteamiento, podemos entender las reglas como normas más objetivas y las idiosincrasias como normas más subjetivas.

Como Baker (1998: 164) o Hermans (1999: 75) señalan, lo que hace Toury, básicamente, es recoger el conocido dualismo langue frente a parole (en términos de Saussure, 1922) o competence frente a performance (en términos de Chomsky, 1965) e introducir un nivel intermedio que le permita investigar aquello que es típico, y no sólo aquello que es o que puede ser. Es decir, las normas operan en un nivel intermedio entre la competencia (competence) y la actuación (performance), donde competencia se refiere al conjunto de opciones que el traductor tiene a su disposición en un determinado contexto y actuación al subconjunto de opciones que el traductor verdaderamente selecciona en la vida real. En este marco, las normas constituirían un nuevo subconjunto de opciones por las que el traductor se decanta de forma regular en un determinado contexto sociohistórico.

Como antes se apuntaba, el concepto de norma no es exclusivo de los estudios descriptivos y se trata de una noción común en el campo general de las ciencias humanas y sociales. Por otra parte, pese a lo que el término normas podría sugerir, aquí se entienden (al igual que hace Toury) no como un conjunto de opciones de índole prescriptiva, sino como una categoría para el análisis descriptivo. En el marco de la traducción, por otro lado, el citado autor considera que el concepto de conducta gobernada por normas se aplica a todo tipo de traducción, no sólo a la literaria, a la filosófica o a la bíblica (1995: 57), y podríamos añadir que también a toda modalidad general de traducción, justificando así la aplicación de dicho concepto al ámbito de la traducción audiovisual. 


\subsection{Las normas como constructo social}

Las normas, lógicamente, no surgen de la nada. Según escribe Toury (1995: 55), los individuos adquirimos normas durante el proceso de socialización, proceso que, por otro lado, no está exento de sanciones. En el seno de una comunidad, las normas sirven también como criterios según los cuales evaluamos instancias reales de comportamiento. Es obvio, añade el autor, que asumir la existencia de normas sólo tiene sentido en aquellas situaciones en las que son posibles diferentes tipos de comportamientos (la traducción es un caso claro), dándose además la condición de que la selección entre ellas no sea aleatoria. Así, siempre y cuando una norma sea realmente activa y efectiva, podremos distinguir una regularidad de conducta ante situaciones recurrentes del mismo tipo. Como apunta Baker (1998: 164), en el marco de la traducción, pues, las normas serán las soluciones que los traductores seleccionan de forma regular en un determinado contexto sociohistórico.

Toury (1995: 55-56) entiende las normas como elementos clave en el establecimiento y mantenimiento del orden social y expresa que, de no existir éstas, se produciría una variación libre extrema, ya que todas las decisiones se tomarían de forma individual. Ello no significa que no sea posible encontrar una conducta que no se ciña a las normas vigentes (non-normative behaviour). Efectivamente, como Hermans señala, el hecho de no cumplir con una norma en casos particulares no invalida dicha norma (1991: 162). Ahora bien, Toury advierte, optar por un tipo de conducta que se desvíe de la norma en vigor supondrá normalmente el pago de un precio (1995: 55). Dicho precio, prosigue el autor, puede ser tan bajo como la mera necesidad de someter el producto final a una revisión, o tan grave que llegue a afectar al prestigio del traductor, posibilidad por la que en la práctica real la conducta no-normativa suele ser una excepción. Por otro lado, no hay que obviar que cuando consideramos en retrospectiva ciertas actuaciones de este tipo podemos comprobar que algunas han llegado a implementar cambios en el sistema, por lo que su estudio constituye también un interesante campo (1995: 64).

\subsection{Características de las normas}

Toury (1995: 61-63) no niega la importancia de la dificultad que rodea a la detección de las normas de traducción, que básicamente reside en dos rasgos inherentes a la noción de norma y, por tanto, no exclusivos de los Estudios sobre la Traducción, que son su especificidad sociocultural y su inestabilidad. Por un lado, una norma no necesariamente afecta a todos los sectores de una sociedad. De forma similar, tampoco será indefectiblemente de aplicación entre culturas. Por otra parte, las normas, como todo aspecto cultural, son entidades inestables y cambiantes por naturaleza, algo que para autores como Díaz Cintas (2005: 14) nos libera de las posturas prescriptivas y autoritarias propias de corrientes teóricas anteriores. Como Toury añade, en ocasiones, las normas cambian de manera rápida; en otras, se muestran más duraderas, con lo que el proceso de cambio se alarga. De hecho, no es extraño encontrar en una sociedad tres tipos de normas compitiendo entre ellas: (1) las normas que 
dominan el centro del sistema y, por consiguiente, la conducta traductora del grupo dominante, (2) los restos de normas anteriores y (3) los rudimentos de otras nuevas suspendidas en la periferia.

Aludiendo a Mukarovský (1970 [1936]), Hermans (1999: 75) menciona un aspecto de las normas que de alguna manera podemos relacionar con su carácter dinámico. En concreto, el autor dice que diferentes espectadores o lectores pueden evaluar un mismo trabajo de manera distinta dependiendo de las normas que sobre éste proyecten, las cuales no necesariamente serán las inicialmente seguidas por el autor. A partir de esto, presumo que la proyección de distintas normas sobre una determinada traducción por parte de distintos receptores puede tener un efecto similar al de las diferentes interpretaciones que un texto puede tener dependiendo del distinto conocimiento previo del mundo que los diversos receptores posean.

Según relata Hermans (1999: 80), el término norma se refiere tanto a una regularidad de conducta (es decir, a un patrón recurrente) como al mecanismo subyacente que da cuenta de esta regularidad. Dicho mecanismo es una entidad psicológica y social que media entre el individuo y el colectivo, entre las intenciones, elecciones y acciones individuales y las creencias, valores y preferencias colectivas. Las normas guardan relación con la interacción entre personas, sobre todo con el grado de coordinación necesario para la coexistencia continuada y más o menos armónica entre los miembros de un grupo. Las normas cumplen una función social reguladora debido a que contribuyen a la estabilidad de las relaciones interpersonales al reducir la incertidumbre, puesto que permiten predecir la conducta generalizando a partir de la experiencia pasada y realizando proyecciones respecto a futuras situaciones similares. Es precisamente ese carácter predictivo de las normas el que, a mi entender, otorga poder de predicción a los resultados de las investigaciones de índole descriptiva.

De modo similar, Hermans (1999: 72) entiende la traducción como acción social. Para el autor, aprender a traducir conlleva un proceso de socialización, ya que significa aprender a manejar (y quizás a manipular) las normas de traducción. En este marco social podemos entender que una comunidad adopte ciertas normas distintas a las de otro grupo. Consecuentemente, la noción de lo correcto (en términos de conducta, uso lingüístico o traducción) constituye un constructo social, cultural e ideológico, con lo que la corrección en traducción es un concepto relativo desde el punto de vista lingüístico, social, político e ideológico (1999: 83-85). Ahora bien, prosigue el autor (1999: 95), dado que la noción de corrección que se deriva a partir de las normas posee una naturaleza cultural, sí que es posible afirmar que una traducción correcta será aquella que responda a las expectativas sobre lo que ha de ser una buena traducción. Por otra parte, esto último podría invitar a una interesante reflexión sobre la docencia de la traducción en contextos multilingües (nuestras aulas, en las que cada año comparten pupitre estudiantes nacionales y extranjeros, constituyen un excelente banco de pruebas).

Según señala Baker (1998: 165), el concepto de norma otorga prioridad al texto meta y, como apunta Hermans (1995: 217), ha reemplazado de forma efectiva al concepto de equivalencia como término operativo en los Estudios sobre la Traducción. Por otra parte, como nos recuerda Baker (1993: 240), el concepto de norma 
asume que el objeto principal de análisis en los Estudios sobre la Traducción no es una traducción individual, sino un corpus coherente de textos traducidos ${ }^{6}$.

Por último, respecto al estudio de las normas de traducción, Toury (1995: 65) sugiere que las normas no son directamente observables (son abstracciones) y que sólo los productos se encuentran disponibles, habiendo dos fuentes principales a partir de las cuales reconstruir las normas:

- Textuales: los mismos textos traducidos para cualquier tipo de norma o los inventarios analíticos de traducción para diversas normas preliminares.

- Extratextuales: formulaciones semiteóricas o críticas, como teorías prescriptivas de traducción, declaraciones de los traductores, editores o cualquier otra persona relacionada con la actividad, valoraciones críticas de traducciones individuales, la actividad de un traductor o de una escuela de traductores, etc.

El hecho de que las normas constituyan abstracciones parece plantear dudas respecto a cómo ofrecer clasificaciones de las mismas. En este sentido, Hermans (1999: 85) coincide con Toury en la imposibilidad de observar las normas de forma directa. Para el autor, la formulación de una norma y la propia norma son dos cosas distintas. Dicho de otro modo, trazar regularidades en distintos textos y entenderlas como el resultado de las decisiones y elecciones de un traductor no nos dice por qué se tomaron dichas decisiones y elecciones. Por su parte, Nord (1991: 103-105) coincide en que las normas (la autora habla de convenciones) no están formuladas de forma explícita. Por ello, la autora propone algunas fuentes que considera más o menos útiles para localizar dónde (y cómo) operan: (1) el análisis de traducciones existentes, (2) la crítica de traducciones, (3) planteamientos teóricos, (4) las opiniones de los usuarios y (5) la comparación multilingüe de traducciones.

Por mi parte, pienso que es del todo posible identificar tendencias (término que definiré más adelante) e incluso normas a partir de investigaciones más o menos extensas y encontrar una explicación pragmática y cultural que pueda justificar su uso y presencia en distintos productos audiovisuales. Por todo ello, en líneas generales podemos asumir las normas como una categoría válida para el análisis descriptivo de la traducción audiovisual. Como bien expresa Díaz Cintas (2005: 14):

En lugar de lanzar ideas abstractas sin datos empíricos que las fundamenten, o de inventar ejemplos ad hoc para ilustrar un determinado punto que conviene al

\footnotetext{
${ }^{6}$ Esto parece ser ignorado en ocasiones en las que se nos presentan presuntas normas a partir de estudios de casos que se reducen a un único filme. En el polo opuesto, proyectos como Forlixt 1, The Forlì Corpus of Screen Translation, llevado a cabo por miembros del Dipartimento di Studi Interdisciplinari su Traduzione, Lingue e Culture de la Universidad de Bolonia (Forli), suponen desde 2003 un buen ejemplo de la envergadura que puede llegar a alcanzar una investigación destinada, entre otras cosas, a la búsqueda de regularidades y normas operativas. El corpus de dicho proyecto está formado actualmente por 99 filmes (para más detalles véase Valentini y Linardi, 2009). En todo caso, de nuevo parece razonable establecer (si es que es posible) cuantitativa y, por tanto, objetivamente en qué momento podemos hablar con propiedad de normas, dado que de momento parece ser una cuestión que responde a criterios subjetivos y por ende arbitrarios, algo que sin duda resta validez a la teoría.
} 
investigador de turno, lo que ahora se sugiere es llevar a cabo un mapping de lo que verdaderamente ocurre cuando se traduce, para evitar caer en la teorización más absoluta. Sólo a partir de ejemplos reales, que existen y se han visto integrados en la sociedad de acogida, podemos extraer conclusiones que nos hagan avanzar en el conocimiento de la traducción. Y las normas son, precisamente, los útiles que nos ayudan en esta tarea.

\subsection{Clases de normas de traducción}

El paradigma normativo establece una teoría emergente que, en el presente estadio, está prácticamente consolidada. No es mi pretensión realizar en el presente artículo una extensa descripción teórica de los conceptos de convención (convention), norma (norm), regla (rule) o ley (law). Para mis propósitos, será suficiente con explicar algunos términos que juzgo capitales. Enumeraré en primer lugar las clases de normas de traducción propuestas por Toury (el precursor), para seguidamente nombrar las formuladas por otros autores y finalizar con las normas que se han sugerido desde el ámbito de la traducción audiovisual.

\subsubsection{Las normas de Toury}

Toury (1995: 56-61) distingue tres tipos de normas de traducción, que son las que determinan el tipo y el alcance de la equivalencia que las traducciones manifiestan:

1. La norma inicial (initial norm). Esta norma tiene que ver con la elección básica entre la adhesión a los requerimientos y normas del texto origen o a los del texto meta. En otros términos, la norma inicial constituye la orientación bien hacia el sistema origen, bien hacia el sistema meta, teniendo en cuenta que existe un continuo entre ambas orientaciones. Según lo entiendo, es posible conectar esta norma inicial con las nociones de extranjerización (adecuación) y de familiarización (aceptabilidad).

2. Las normas preliminares (preliminary norms). Estas normas tienen que ver con dos tipos de consideraciones a menudo conectadas entre ellas. Por un lado, la existencia de una política de traducción ${ }^{7}$ y, por otro, la de unas consideraciones que afectan al grado de tolerancia existente con respecto a la traducción indirecta (es decir, a la traducción de una traducción).

3. Las normas operacionales (operational norms). Son normas que dirigen las decisiones que se toman durante el mismo acto de traducción, gobernando aquello que probablemente permanecerá invariable y aquello que variará. Estas normas se dividen a su vez en dos subgrupos: las normas matriciales

\footnotetext{
${ }^{7}$ Se hace difícil separar el término política de un planteamiento prescriptivo.
} 
(que gobiernan la existencia del material de la lengua meta correspondiente que se pretende para sustituir al material de la lengua origen, su ubicación en el texto y la segmentación textual) y las normas lingüístico-textuales (que gobiernan la selección del material para formular el texto meta o con el que reemplazar el material textual y lingüístico original).

\subsubsection{Crítica al modelo de Toury y otras propuestas}

\section{A. Crítica}

No podemos obviar que algunos planteamientos de Toury no han escapado a la crítica. A continuación rescataré algunas de las apreciaciones de Hermans, uno de los autores que han tratado de ir un poco más allá de lo postulado por el autor israelí, por coincidir en líneas generales con dichas valoraciones. Tras ello, me haré también eco de las consideraciones más recientes de Díaz Cintas, otro de los autores que han detectado aspectos criticables en este modelo.

Según Hermans (1999: 76-77, 79-80), el aspecto más problemático tanto conceptual como terminológicamente de la teoría de Toury lo constituyen las nociones excluyentes de adecuación frente a aceptabilidad comprendidas en la norma inicial. Hermans propone como alternativa la sustitución de la oposición aceptable frente a adecuado por la de orientado hacia el sistema meta (target-oriented) frente a orientado hacia el sistema origen (source-oriented). Sobre esto último, quisiera precisar que, desde mi punto de vista, en lugar de hablar de oposición absoluta, me parece más acertado hablar de dos extremos de un continuo. Es más, en un producto audiovisual (una comedia de situación, por ejemplo) podemos encontrar una combinación de ambos métodos.

Hermans concreta que sería incluso mejor no concebir la norma inicial como una elección forzosa entre sólo dos polos, sino como una norma en la que confluyan factores múltiples como, por ejemplo, la visión que se tenga del texto origen, si dicho texto u otros similares se han traducido con anterioridad, si la traducción se hace para su importación o exportación, de qué lengua es nativa la persona que se encarga de llevarla a cabo o para qué audiencia o con qué propósito se realiza (información que, en resumidas cuentas, constituiría un completo encargo de traducción).

En definitiva, Hermans, con un innegable trasfondo funcionalista, pone de manifiesto una serie de aspectos que han de ser, a mi juicio, considerados a la hora de trabajar con normas de traducción. Como bien dice el autor, si consideramos la traducción como una actividad sociocultural, tratar de conceptualizarla en términos de una elección a lo largo de un único eje carece de mucho sentido.

Un aspecto más que Hermans critica es que Toury no haya explorado con mayor profundidad el lado teórico de las normas de traducción, puesto que en la práctica Toury enfoca la cuestión desde el punto de vista del traductor y concibe las normas como restricciones (constraints), ignorando su papel como plantillas (templates) que ofrecen soluciones preelaboradas (ready-made solutions) a ciertos tipos de problemas. Dicho de otro modo, Hermans entiende que, cuando un traductor se decanta 
por una determinada opción, realiza una elección a partir de un inventario de soluciones disponibles. Elegir una opción significa excluir las alternativas, si bien éstas permanecen latentes como almacén de posibilidades futuras (1999: 88). Esta visión, me parece, justifica aquellos estudios que anhelan identificar una serie de tendencias o de normas de traducción (audiovisual) que contribuyan al avance de la disciplina.

Además de en Hermans, podemos encontrar otra fuente crítica en Díaz Cintas (2005: 14-15), quien evidencia una serie de limitaciones del concepto de norma, como por ejemplo: (1) que el carácter cambiante de las normas, si bien facilita su análisis en textos de épocas pasadas, dificulta tal labor en textos actuales, precisamente por ese cambio constante, ante lo que el autor propone sustraer prioridad a la dimensión histórica ${ }^{8},(2)$ que su aplicación parece producir mejores resultados cuando se analizan de modo contrastivo varios filmes en lugar de uno solo, algo que a su vez se traduce en investigaciones ingentes que pueden requerir una labor de equipo, restando protagonismo a la actividad investigadora individual ${ }^{9}$; o (3) el peligro de generalización que supone el deseo de identificar normas que han actuado durante un periodo demasiado extenso y la posibilidad de que siempre se puedan cuestionar tales conclusiones generales al tratarse de un concepto que, llevado a sus extremos, podría equiparar el nivel normativo con el individual, es decir, cada norma con una realización por persona. Frente a este último obstáculo, Díaz Cintas sugiere restar expectativas y amplitud a las normas mediante su búsqueda en corpora más homogéneos y abarcables (¿cuántos?), tarea para la que la traducción audiovisual resulta ideal. Pensemos que, como nos recuerda el autor, en este ámbito las normas pueden venir aplicadas no por los traductores de manera individual (operacionales), sino por otros agentes como los estudios, las distribuidoras, los directores de doblaje y ajustadores, etc. (preliminares), algo que, a priori, parece facilitar la observación de regularidades normativas si centramos el análisis en, por ejemplo, los productos comercializados por una determinada cadena de televisión.

Ahora bien, Díaz Cintas entiende las anteriores como objeciones menores que no han de menoscabar la importancia del concepto que nos ocupa. Como el autor refleja, el éxito del concepto de norma reside en el hecho de que proporciona un objetivo claro al investigador y le dirige hacia lo que tiene que encontrar. El objetivo, pues, queda claro, y se evita caer en una búsqueda errática. Más que pretender evaluar la equivalencia de dos textos traducidos, la importancia radica en poner de manifiesto las razones por las que se ha llegado a la misma y el significado que ello supone en el marco sociocultural en que se ha llevado a cabo la traducción. De nuevo la duda que me viene al pensamiento es si puede resultar posible que este comportamiento nos conduzca a un enrocamiento de la disciplina.

Por último, quisiera añadir una crítica a las ya expuestas, la cual ya se ha esbozado en párrafos anteriores. Existe un acuerdo claro sobre qué son las normas y

\footnotetext{
${ }^{8}$ En todo caso, cabe suponer que habrá ciertas normas para cuyo cambio sea preciso un periodo de tiempo ciertamente considerable, algo que quizá minimice el efecto de dicho carácter cambiante.

${ }^{9}$ Este argumento resulta válido para apoyar la conveniencia de las tendencias como estadio intermedio entre las estrategias y las normas, puesto que posibilitan dicha investigación individual o en grupos reducidos.
} 
sobre su utilidad para el estudio descriptivo de la traducción, al menos en lo que concierne a las normas operacionales. Ahora bien, definir de manera clara en qué momento una estrategia recurrente deja de serlo y se convierte en una norma es una cuestión pendiente de resolver.

\section{B. Otras propuestas}

A continuación, recogeré de modo esquemático algunas de las propuestas que han tratado de completar los posibles vacíos dejados por el planteamiento de Toury (ya que éste no va más allá del nivel microtextual, algo que puede y debe hacerse, siendo quizás el límite el contexto de cultura ${ }^{10}$ ) y que, por otra parte, reflejan la falta de unanimidad respecto a esta cuestión:

- Como resume Baker (1998: 164-165), en los últimos años una serie de autores han tratado de explorar algunos de los aspectos teóricos de la noción de norma, distinguiendo primero entre normas y convenciones (la segunda no tiene carácter obligatorio y tan sólo expresa una preferencia) y después entre normas constitutivas (constitutive norms) y normas reguladoras (regulatory norms) (las primeras conciernen a aquello que se acepta o no como traducción y las segundas al tipo de equivalencia por el que traductor opta o que consigue).

- Por su parte, Chesterman (1997: 64-70) hila aún más fino y distingue entre las normas de expectativa (expectancy norms) o de producto (que reflejan las expectativas que los receptores de una traducción tienen con respecto a cómo debe ser una traducción) y, a un nivel inferior, las normas profesionales (professional norms), de proceso o de producción (que gobiernan los métodos y las estrategias del proceso traductor). El enfoque de Chesterman es, al igual que el Toury, descriptivo. Son también interesantes las aportaciones de Chesterman y Wagner (2002).

- Nord (1991), quien habla de normas (o convenciones, según su terminología) constitutivas (constitutive) y reguladoras (regulatory). Aparte de estas dos convenciones, en un trabajo posterior de la autora (1997) encontramos una propuesta ampliada. Nord (quien describe las convenciones como regularidades sociales formuladas de manera tácita, no obligatorias y basadas en el conocimiento común y expectativas, 1991: 96) considera el papel que juegan las convenciones en los enfoques funcionalistas de la traducción. La autora (1997: 53) comenta que, en el marco de la teoría del escopo, Reiss y Vermeer se limitan a las convenciones de género. Sin embargo, entiende que hay más tipos de convenciones que han de considerarse en el marco de la traducción funcional (1997: 53-59): las convenciones de género (genre conventions), las convenciones de estilo general (general style conventions), las convenciones de con-

10 Para una completa explicación de dicho contexto véase Marco (2002). 
ducta no-verbal (conventions of nonverbal behaviour) y las convenciones de traducción (translation behaviour).

- Finalmente, no quisiera olvidar la aportación de Rabadán (1991: 56-57), quien, dentro del grupo de las normas operacionales de Toury, añade las normas de recepción, las cuales, teniendo en cuenta el tipo de audiencia que se prevé (según el encargo de traducción), determinan el modo en el que actúa el traductor.

Como es fácilmente deducible, nos encontramos ante un debate terminológico, ya que en varias ocasiones estos autores se refieren a lo mismo si bien emplean etiquetas distintas.

\subsection{Normas en traducción audiovisual}

Partiré del siguiente comentario de Delabastita (1989: 205):

The translation of film, then, constitutes a typical situation where one can expect norms to guide the selection of actual behaviour in each specific historical set of circumstances. As a result of such norms the different possible modes of translation will not all be 'in free variation', as some of them will in the given conditions implicitly or explicitly be judged as 'better' solutions than the others.

Me parece productivo considerar la posibilidad de encontrar normas en cualquier modalidad de traducción audiovisual y en la gestión de cualquier material audiovisual (no sólo en la de los filmes). De hecho, no faltan autores que se hayan ya ocupado de buscar normas en el entorno de la traducción audiovisual. En este punto, me voy a referir al trabajo de Goris (1991 y 1993) y al de Ballester (2001), cuyas respectivas propuestas servirán de ilustración de lo hasta el momento planteado al respecto. De dichas propuestas me interesan, principalmente, los diferentes tipos de normas que los autores proponen. También se han escrito trabajos de investigación y tesis doctorales sobre este asunto (véase Roussou, 2003, Martínez Sierra, 2004 y 2008 b y Martí Ferriol, 2006). Por otro lado, el grupo TRACE, compuesto mayoritariamente por investigadores de las Universidades de León, País Vasco y Valladolid, busca normas de traducción audiovisual en el cine traducido de la España franquista (véase, por ejemplo, el trabajo coordinado por Rabadán, 2000). Del mismo modo, recordemos la alusión al proyecto Forlixt 1 (véase nota 6).

Goris se marca como objetivo realizar un estudio descriptivo del doblaje (si bien el autor no ignora la posibilidad de aplicar sus resultados a la subtitulación) en Francia, con el fin de analizar cómo un sistema meta se apropia de un mensaje internacional (como, por ejemplo, un filme). Para ello, el autor parte de un corpus compuesto por cinco películas. Tras su análisis concluye que, en la traducción de los citados filmes, es posible observar la aparición sistemática de tres normas principales (dos de ellas operacionales) con un poder significativo de influencia en el doblaje (1991: 86-118, 127 y 1993: 173-185): 
- La estandarización lingüística, consistente en la homogeneización de la versión meta.

- La naturalización, consistente en operar sobre la versión meta de forma que se asemeje a una versión origen hecha en la cultura meta (según lo veo, parece posible encontrar puntos de conexión entre esta norma y un método familiarizante o, dicho de otro modo, con la norma inicial de Toury).

- La explicitación, consistente en dotar a la versión meta de mayor claridad y precisión que la versión origen.

Además de estas tres normas principales, Goris introduce dos normas secundarias (también operacionales) que, dada su aparición menos sistemática, tienen una menor influencia sobre la traducción (1991: 119 y 1993: 185-186):

- El mantenimiento de los nombres y oraciones simples del texto origen.

- La conservación de las características específicas del filme, que están en parte determinadas por el género al que éste pertenece.

Basándose en la propuesta metodológica de Lambert y Delabastita y en la aplicación que de ella hace Goris, Ballester lleva a cabo un estudio contrastivo del guión origen y del traducido para el doblaje de la película estadounidense Sangre y Arena (Blood and Sand, Rouben Mamoulian, 1941). Como resultado de dicho estudio, la autora identifica una serie de normas de traducción, tres principales y una secundaria $^{11}$. Veamos primero las principales (2001: 160-197):

- La autocensura, norma (preliminar) de naturaleza eufemística que pretende, además de censurar o suavizar la versión meta desde el punto de vista moral, religioso e ideológico, mejorar la imagen del torero.

- La naturalización (de nuevo, norma inicial), si bien la autora, al analizar el guión y no la película, se centra en el tratamiento de las palabras extranjeras y de las referencias culturales.

- La explicitación (norma operacional), que en el caso del corpus de Ballester se traduce en la construcción de enunciados más completos desde el punto de vista sintáctico y más precisos semánticamente.

Como norma secundaria, Ballester señala la falta de rigor en la expresión en español (errores sintácticos, ortográficos, de acentuación y de puntuación) ${ }^{12}$.

${ }^{11}$ Como antes se indicaba, cabría cuestionarse si el análisis de un único filme basta para proponer normas. Recordemos los comentarios de Baker y Díaz Cintas sobre la necesidad de analizar un corpus extenso con objeto de identificar normas. De manera adicional, como el mismo Goris reconoce, "Since the análisis is based on a limited corpus of texts, the results should be taken as tentative; the question of the universality of the uncovered norms can only be solved by further research" (1993: 173).

12 Resulta interesante preguntarse si dichos errores son fruto de una elección o simplemente de una preparación deficiente. 


\section{Tendencias de traducción}

Tras ocuparme de los diferentes tipos de normas que distintos autores han planteado, tanto desde la perspectiva más general de la traducción como desde la más concreta de la traducción audiovisual, es ahora momento de realizar una precisión respecto a las normas que se derivan directamente de la actuación de los traductores (es decir, las operacionales).

A mi juicio, y partiendo de lo expuesto con anterioridad en el presente trabajo, en el tratamiento de las normas hay dos cuestiones preliminares que es necesario plantearse. La primera nos sugiere, irremediablemente, una labor que sobrepasa el alcance del presente artículo y que podría, por sí sola, constituir el objeto de análisis ya no de otro artículo, sino de un proyecto de investigación de envergadura que contara con mayor tiempo, personal y, por qué no decirlo, fondos. En concreto, la pregunta, ya planteada en páginas anteriores, es: ¿a partir de qué cantidad de casos podemos hablar de norma? Se ha hablado de traductores que optan por una solución de forma regular. ¿Pero dónde está la línea entre actuación puntual y comportamiento regular? También se ha aludido al análisis de corpora extensos, ¿pero cómo se definen cuantitativamente dichos corpora? Como digo, la respuesta queda fuera del alcance de este artículo, y sería necesaria una reflexión y seguramente una investigación más extensas para poder dar cuenta de esta curiosidad científica. Sin embargo, sí creo estar en situación de poder afirmar que es preciso, al menos conveniente, afrontar dicha cuestión, y como un posible primer paso propongo hablar de tendencias (regularidades) detectadas en la traducción de un producto audiovisual o incluso de un texto escrito ${ }^{13}$, algo posible no sólo a partir de los cambios observados entre los dos textos, sino también a partir de aquello que ha permanecido inalterado. Dichas tendencias suponen un primer acercamiento a partir del cual ampliar el número de capítulos de una serie o de filmes examinados y poder así llegar a comprobar cuáles de las tendencias que se presentan muestran un carácter claramente recurrente en su traducción de la serie (con lo que ya podríamos empezar a hablar de normas) y cuáles de ellas tienen una naturaleza más puntual (con lo que se quedarían en la aplicación frecuente de una determinada estrategia ${ }^{14}$ ).

La segunda cuestión es la siguiente: ¿cuál es el estadio previo a una norma? En parte, ya he contestado a esta pregunta en el párrafo anterior. Según lo entiendo, partimos de una serie de decisiones individuales que un traductor toma o, dicho de otro modo, de una serie de estrategias de traducción. Una vez observamos que un mismo traductor (o equipo de traductores) emplea de forma regular una determinada estrategia en la traducción de casos similares (siempre que el proceso se desarrolle bajo unos mismos parámetros socioculturales), podremos empezar a considerar la posi-

\footnotetext{
13 Recordemos que el concepto de conducta gobernada por normas se aplica a todo tipo y modalidad de traducción.

14 Como nos recuerda Valdés (1999: 147), "una estrategia traductora es el resultado de las decisiones tomadas por el traductor durante todo el proceso de traducción". Véase también Hurtado (2001), quien distingue entre estrategias y técnicas.
} 
bilidad de una tendencia traductora (operacional en este caso). Cuando constatemos la actuación recurrente de una determinada tendencia nos será posible pensar en una norma de traducción aunque, como ya se ha advertido, no se procederá aquí a cuantificar el número de reiteraciones de un caso necesarias para estar en disposición de hablar de normas. La Figura 1 plasma de forma gráfica el proceso.

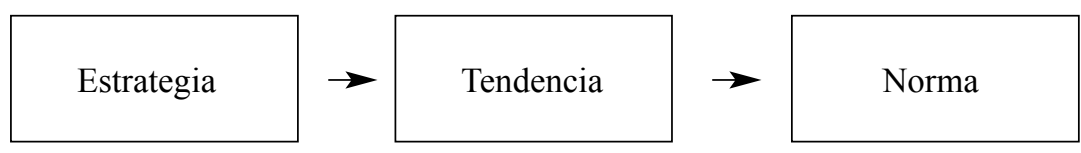

\section{Precisiones finales}

En este trabajo he pretendido, fundamentalmente, llevar a cabo dos labores: por un lado, resumir la teoría de las normas con objeto de ofrecer al lector un retrato completo y útil de la misma y, por otro, poner sobre la mesa dos cuestiones que considero han de plantearse y que resumo a continuación.

La primera cuestión para la reflexión afecta al paradigma descriptivo en su conjunto. En líneas generales, en la última década la investigación en el campo de la traducción audiovisual ha sido fundamentalmente descriptiva, tratándose de un ámbito que lleva por tanto años nutriéndose de estudios descriptivos destinados a dibujar el mapa de la disciplina, algo que es necesario hacer. Ahora bien, una vez dicho mapa esté completo, ¿cuál será el siguiente paso? ¿Aplicaremos la ley del péndulo y regresaremos a las actitudes prescriptivas? Asimismo, ¿basta con describir para que avance la disciplina o, además de explicar qué se ha hecho habría que indicar cómo debería haberse hecho y cómo habrá de hacerse en un futuro? Y, de ser así, ¿quién debería encargarse de tal indicación? Por otro lado, y principalmente pensando en la formación de nuevos traductores, parece razonable asumir que la docencia posee un importante, si bien no necesariamente único, componente prescriptivo ${ }^{15}$. Por tanto, ¿no es posible un término medio en el que descripción y prescripción no sean excluyentes sino complementarias en el ámbito de la investigación, al igual como seguramente ya ocurre en muchas aulas ${ }^{16}$ ?

15 Por un lado, recordemos que, como antes se recogía, para autores como Díaz Cintas (2005) el modelo descriptivo posee ciertas características que lo alejan del ámbito docente. Por otra parte, sin embargo, es posible identificar ciertos aspectos de dicho paradigma que pueden ser perfectamente aplicables a la didáctica de la traducción, como por ejemplo la naturaleza predictiva de las normas y el uso sugerido por Hermans (1999) de las mismas como plantillas de soluciones a problemas.

16 Autores como Chaume comentan que "hemos recurrido al paradigma polisistémico por ser el único hasta la fecha que se ha mostrado lo suficientemente sistemático y sólido como para ofrecer las bases metodológicas necesarias para emprender una investigación rigurosa", y opinan que "no debería ser este el único referente científico, puesto que el estudio de las normas de traducción tiene un horizonte limitado" (2004: 94). 
En segundo lugar, se ha presentado la cuestión de las tendencias como paso intermedio entre estrategias y normas y como útil de trabajo. Soy consciente de que el término tendencia no resuelve el problema cuantitativo, ya que igualmente se puede cuestionar a partir de qué momento una estrategia deja de serlo y se convierte en tendencia y cuándo una tendencia deja de serlo y se convierte en norma. Aún así estimo que puede ser una etiqueda extremadamente útil para catalogar los resultados de aquellas investigaciones (incluso las individuales) que quieran denotar su naturaleza descriptiva y que deseen dejar patente su intención de ir más allá de la detección de unas cuantas estrategias recurrentes, sin por ello hablar de normas de manera dúctil e incluso osada y sin descuidar por tanto la prudencia que el rigor científico exige.

No son pocas las voces que solicitan, por otra parte con todo derecho, que los Estudios sobre la Traducción en general y la Traducción Audiovisual en particular sean consideradas disciplinas o campos respetados y académicamente bien considerados (especialmente en el segundo caso, sobre el que aún hoy sobrevuela la creencia de que constituye la cara desenfadada de la traducción). Sin embargo, ¿cómo podemos demandar el citado respeto si en ocasiones desde el seno del propio ámbito se descuida el rigor preciso para obtener dicho reconocimiento?

Para finalizar, soy consciente de que no faltarán los lectores que se pregunten qué problema supone utilizar el término norma en cualquier estudio descriptivo, independientemente del número de casos analizados. ¿Por qué proponer otro término? Por mi parte, ya he explicado los motivos, pero quisiera añadir uno más en forma de pregunta: si para hablar de normas basta con analizar dos o tres películas, ¿qué sentido tiene la investigación basada en corpus? ¿A qué han dedicado sus esfuerzos aquellos grupos de investigación que lleva años analizando filmes en busca de regularidades?

\section{Referencias bibliográficas}

Baker, Mona, «Corpus Linguistics and Translation Studies. Implications and Applications», en: Baker, Mona et al. (eds.), Text and Technology: In Honour of John Sinclair. Ámsterdam: John Benjamins 1993, 233-250.

- (ed.), Routledge Encyclopedia of Translation Studies. Londres / Nueva York: Routledge 1998.

Ballester, Ana Rosa, Traducción y nacionalismo. La recepción del cine americano en España a través del doblaje (1928-1948). Granada: Comares 2001.

Cattrysse, Patrick, «The study of film adaptation: a state of the art and some 'new' functional proposals», en: Eguíluz, Federico et al. (eds.), Trasvases Culturales: Literatura, Cine, Traducción. Vitoria: Euskal Herriko Unibertsitatea 1994, 37-55.

Chaume, Frederic, Proyecto docente presentado en la Universitat Jaume I 2001.

Chesterman, Andrew, «From 'Is' to 'Ought': Laws, Norms and Strategies in Translation Studies», Target 5:1 (1993): 1-20.

—, Memes of Translation. Ámsterdam / Filadelfia: John Benjamins 1997.

Chesterman, Andrew y Emma Wagner, Can Theory Help Translators? A Dialogue Between the Ivory Tower and the Wordface. Manchester: St. Jerome 2002. 
Chomsky, Noam, Aspects of the Theory of Syntax. Cambridge (MA): The MIT Press 1965.

Delabastita, Dirk, «Translation and Mass-Communication: Film and TV Translation as Evidence of Cultural Dynamics», Babel 35:4 (1989): 193-218.

Díaz Cintas, Jorge, «Teoría y traducción audiovisual», en: Zabalbeascoa, Patrick et al. (eds.), La traducción audiovisual: Investigación, enseñanza y profesión. Granada: Comares 2005, 9-21.

Fawcett, Peter, «The Manipulation of Language and Culture in Film Translation», en Calzada Pérez, María (ed.), Apropos of Ideology. Translation Studies on Ideology - Ideologies in Translation Studies. Manchester: St. Jerome 2003, 145-163.

Goris, Olivier, À la recherche de normes pour le doublage. État de la question et propositions pour une analyse descriptive. Tesina presentada en la Universidad Católica de Lovaina 1991.

—, «The Question of French Dubbing: Towards a Frame for Systematic Investigation», Target 55:2 (1993): 169-190.

Hermans, Theo (ed.), The Manipulation of Literature: Studies in Literary Translation. Londres / Sydney: Croom Helm 1985.

—, «Revisiting the Classics. Toury’s Empiricism Version One», The Translator 1:2 (1995): 215-223.

-, Translation in Systems. Descriptive and System-oriented Approaches Explained. Manchester: St. Jerome 1999.

Holmes, James S., The Name and Nature of Translation Studies. Ámsterdam: Department of General Literary Studies, Translation Studies Section 1972.

Hurtado, Amparo, Traducción y traductología. Madrid: Cátedra 2001.

Ivarsson, Jan, Subtitling for the Media. A Handbook of an Art. Estocolmo: Transedit 1992.

Karamitroglou, Fotius, Towards a Methodology for the Investigation of Norms in Audiovisual Translation. Ámsterdam: Rodopi (St. Jerome) 2000.

Levý, Jirí, «Translation as a Decision Process», en: To Honor Roman Jakobson, vol. 2. La Haya / París: Mouton 1967, 1171-1182.

Marco, Josep, El fil d'Ariadna. Anàlisi estilística i traducció literaria. Vic: Eumo 2002.

Martí Ferriol, José Luis, Estudio empírico y descriptivo del método de traducción para doblaje y subtitulación. Tesis doctoral presentada en el Departament de Traducció i Comunicació de la Universitat Jaume I 2006.

Martínez Sierra, Juan José, Estudio descriptivo y discursivo de la traducción del humor en textos audiovisuales. El caso de Los Simpson. Tesis doctoral presentada en el Departament de Traducció i Comunicació de la Universitat Jaume I 2004.

-, «The Contact between Cultures and the Role of Translation and the Mass Media», Translation Journal 12:1 (2008a).

-, Humor y traducción. Los Simpson cruzan la frontera. Castellón: Universitat Jaume I 2008b.

Mukarovský, Jan, Aesthetic Function, Norm and Value as Social Facts. Suino, M. (trad.). Ann Arbor: University of Michigan 1970 [1936].

Nord, Christiane, «Scopos, Loyalty and Translational Conventions», Target 3:1 (1991): 91109.

—, Translating as a Purposeful Activity. Manchester: St. Jerome 1997.

Popovič, Anton, «The Concept 'Shift of Expression' in Translation Analysis», en: Holmes, James S. (ed.), The Nature of Translation. Essays on the Theory and Practice of Literary Translation. La Haya / París: Mouton, Slovak Academy of Sciences 1970.

Rabadán, Rosa, Equivalencia y traducción: problemática de la equivalencia translémica inglés-español. León: Publicaciones de la Universidad de León 1991. 
—, (coord.), Traducción y censura inglés-español: 1939-1985. Estudio preliminar. León: Publicaciones de la Universidad de León 2000.

Romero Fresco, Pablo, «Subsording travels to Europe: the D’Artagnan project to standardise SDH across Europe», en: Anna Matamala y Pilar Orero (eds.), Digital Subtitles. Berlin: Peter Lang 2009.

Saussure, Ferdinand de, Cours de linguistique générale. París: Éditions Payot 1922.

Toury, Gideon, Descriptive Translation Studies and Beyond. Ámsterdam/Filadelfia: John Benjamins 1995.

Valdés, Cristina, La traducción publicitaria como acto de comunicación intercultural. Tesis doctoral presentada en el Departamento de Filología Anglogermánica y Francesa de la Facultad de Filología de la Universidad de Oviedo 1999.

Valentini, Cristina y Sabrina Linardi, «Forlixt 1: A multimedia database for AVT research», Intralinea (2009). 